\title{
Unilateral follicular variant of papillary thyroid carcinoma with unique KRAS mutation in struma ovarii in bilateral ovarian teratoma: a rare case report
}

Boban Stanojevic ${ }^{1,2^{*}}$, Radan Dzodic 3,4, Vladimir Saenko ${ }^{1}$, Zorka Milovanovic ${ }^{5}$, Vesna Krstevski ${ }^{6}$, Petar Radlovic ${ }^{4}$, Marko Buta ${ }^{4}$, Bozidar Rulic ${ }^{4}$, Lidija Todorovic ${ }^{2}$, Bogomir Dimitrijevic ${ }^{2}$ and Shunichi Yamashita ${ }^{1}$

\begin{abstract}
Background: Struma ovarii (SO) is a rare form of ovarian mature teratoma in which thyroid tissue is the predominant element. Because of its rarity, the differential diagnosis between benign and malignant $\mathrm{SO}$ has not been clearly defined. It is believed that malignant transformation of SO has similar molecular features with and its prognosis corresponds to that of malignant tumors originating in the thyroid.

Case presentation: We report 35-year-old woman with bilateral ovarian cysts incidentally detected by ultrasound during the first trimester of pregnancy. Four months after delivery of a healthy child without complication she was admitted to the hospital for acute abdominal pain. Laparoscopic left adnexectomy was performed initially in a regional hospital; right cystectomy was done later in a specialized clinic. Intraoperative frozen section and a final pathology revealed that the cyst from the left ovary was composed of mature teratomatous elements, normal thyroid tissue (>50\%) and a non-encapsulated focus of follicular variant of papillary thyroid carcinoma (PTC). Normal and cancerous thyroid tissues were tested for BRAF and RAS mutations by direct sequencing, and for RET/PTC rearrangements by RT-PCR/Southern blotting. A KRAS codon 12 mutation, the GGT $\rightarrow$ GTT transversion, corresponding to the Gly $\rightarrow$ Val amino acid change was identified in the absence of other genetic alterations commonly found in PTC.

Conclusion: To the best of our knowledge, this is the first time this mutation is described in a papillary thyroid carcinoma arising in struma in the ovarii. This finding provides further evidence that even rare mutations specific for PTC may occur in such tumors. Molecular testing may be a useful adjunct to common differential diagnostic methods of thyroid malignancy in SO.
\end{abstract}

Keywords: Struma ovarii, Follicular variant of papillary thyroid carcinoma, KRAS mutation

\footnotetext{
* Correspondence: boban.stanojevic@hotmail.com

${ }^{1}$ Nagasaki University Graduate School of Biomedical Sciences, 1-12-4

Sakamoto, Nagasaki, 852-8523, Japan

2Laboratory for Radiobiology and Molecular Genetics "Vinca", Institute of

Nuclear Sciences, University of Belgrade, Mike Petrovica Alasa12-14, P.O. Box

522, 11000, Belgrade, Serbia

Full list of author information is available at the end of the article
} 


\section{Background}

Struma ovarii (SO) is a rare form of ovarian teratoma where thyroid tissue forms a grossly detectible mass or is the major cellular component $(>50 \%)$. Teratomas comprise approximately $95 \%$ of germ cell tumors of which SO accounts for only $3 \%[1,2]$. The differential diagnosis between benign and malignant SO may be difficult due to the lack of uniform diagnostic criteria [3]. The real incidence of malignancy in SO is unclear because of its rarity but the existing literature indicates that $5 \%-10 \%$ of such tumors are malignant with papillary and less frequently, follicular carcinoma being the most common [1-4]. It is believed that malignant transformation of SO has similar molecular features with and its prognosis corresponds to that of malignant tumors originating in the thyroid $[5,6]$. The molecular pathogenesis of papillary thyroid carcinomas (PTC) is largely associated with genetic alterations in the RET-RASRAF-MAPK pathway usually caused by RET/PTC rearrangements $(13 \%-43 \%)$ or activating point mutations in the $B R A F(29 \%-83 \%)$ or $R A S$-family genes $(0 \%-21 \%)$. These molecular alterations occur cumulatively in up to $70 \%$ of all follicular cell-derived thyroid carcinomas in a nearly mutually exclusive manner [6-9].

\section{Case presentation}

The patient is a 35-year-old woman (gravida 1, para 0, abortus 0 ) who during the first trimester of pregnancy was diagnosed for bilateral ovarian cysts detected incidentally by ultrasound. She had no significant medical or gynecological history before and was clinically and biochemically euthyroid with normal serum TSH level. Four months after delivery of a healthy child without a complication she was admitted to the hospital for acute abdominal pain. Laboratory data showed normal biochemical parameters and complete blood count. The plasma level of CA-125 was slightly elevated to $42.72 \mathrm{U} / \mathrm{ml}(35 \mathrm{U} / \mathrm{mL}$, the upper normal limit). An ultrasound scan showed an enlarged left ovary and cystic masses in both ovaries. The patient was operated in December 2008 in a regional hospital where laparoscopic left adnexectomy was initially performed. Intraoperative frozen section pathology revealed that the cyst was composed of mature teratomatous elements including epidermis, sebaceous glands, adipose, normal thyroid tissue (>50\%) and a $10 \mathrm{~mm}$ non-encapsulated cancerous focus (which was confirmed later to be the FV-PTC on final pathology, Figure $1 \mathrm{a}$ and $1 \mathrm{~b})$ [10-12]. Because of the finding of cancer the surgeon made a decision not to proceed with the right oophorectomy and to transfer the patient to specialized oncology clinic.

The tumor from the left ovary showed immunoreactivity to thyroglobulin and thyroid transcription factor-1 (all antibodies from Dako, Carpinteria, CA, USA, data not shown). For molecular investigation $5 \mu \mathrm{m}$ sections were taken, dewaxed, and lightly stained with hematoxylin and eosin. Normal thyroid tissue and PTC area were manually dissected from 3 serial sections. DNA was extracted using Gentra Puregene Tissue kit (Quiagen, Minneapolis, MN, USA). Total RNA was extracted using Recover All Total Nucleic Acid Isolation Kit (Ambion, Applied Biosystems, Foster City, CA, USA). DNA from the tumor and adjacent benign thyroid tissue was tested by PCR/direct sequencing of genomic DNA for genetic abnormalities that have been described in thyroid cancer originating in the neck, including a portion of BRAF exon 15 and codons 12, 13, 31, 60 and 61 of $\mathrm{K}-, \mathrm{H}$ - and N-RAS. RET/PTC1 and RET/PTC3 rearrangements were detected using reverse transcription-polymerase chain reaction (RT-PCR) followed by Southern blotting. This tumor was found to harbor a KRAS codon 12 mutation, the GGT $\rightarrow$ GTT transversion, corresponding to the Gly $\rightarrow \mathrm{Val}$ amino acid change (Figure 2). No mutations were found in adjacent benign thyroid tissue. All experimental techniques and primer sequences used were essentially as previously described [13].

Case was presented to the multidisciplinary committee at the Institute of Oncology and Radiology of Serbia who decided the patient should undergo reoperation for the right ovarian cyst measuring $80 \mathrm{~mm}$, which was also present at the time of the first surgery. The patient was

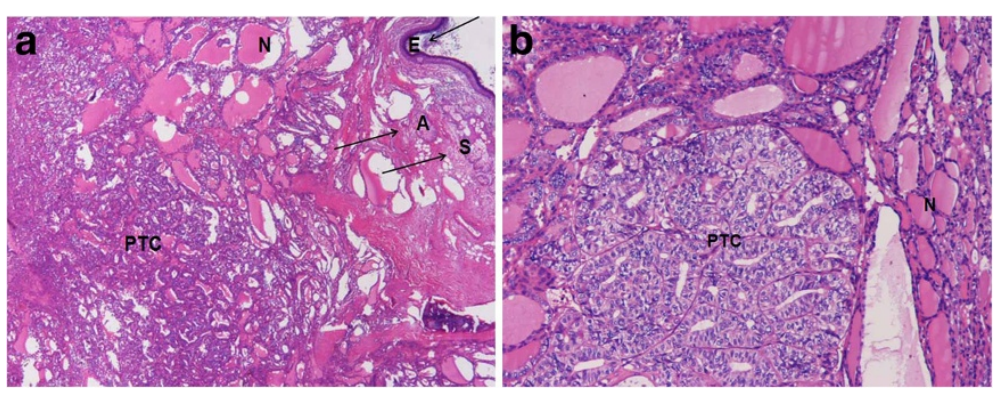

Figure 1 a. Teratoma tissue with mature epidermis (E), sebaceous glands (S), adipose tissue (A) normal (N) and malignant thyroid tissue (PTC) (H\&E 40x). b. Fragment of PTC in SO featuring solid-trabecular and follicular growth patterns (H\&E 100x). 


\section{Glycine (Wild type)}

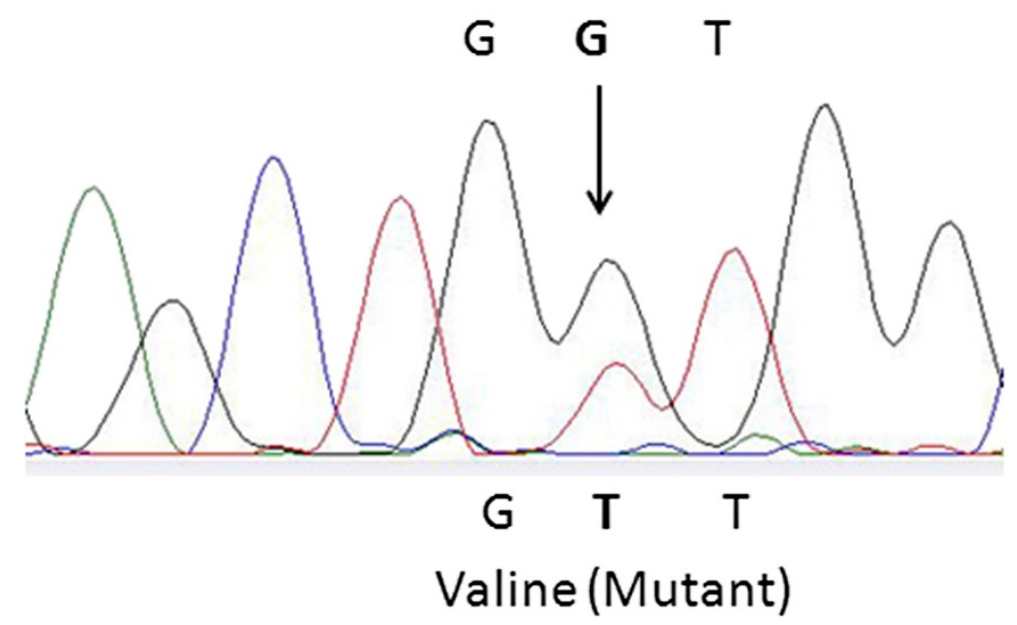

Figure 2 KRAS codon 12 mutation, GGT $\rightarrow$ GTT, corresponding to the Gly $\rightarrow$ Val amino acid change.

operated two months after the initial surgery and after median laparatomy a macroscopically enlarged right ovary was verified to contain two cysts. The larger cyst

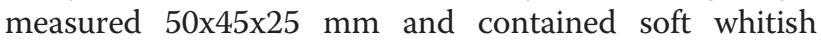
material. Histology report confirmed benign ovarian cyst with no evidence of other abnormality. An intraoperative frozen section and final pathology of the second smaller cyst $30 \times 25 \times 20 \mathrm{~mm}$ in size revealed mature cystic teratoma with benign thyroid tissue. Other tissue components included hair, adipose tissue, cartilage, bone and skin. Both cysts were removed and the right ovary was preserved. Multiple biopsies of the greater omentum, iliac lymph nodes, peritoneum above the urinary bladder and cul de sac showed no signs of malignant spread. Postoperative thyroid function blood test showed normal TSH, calcitonin, thyroglobulin and thyroglobulin antibody levels. Neck ultrasonography revealed a solitary thyroid nodule $(6 \times 4 \mathrm{~mm})$ located caudally in the left lobe without signs of enlarged central and jugulo-carotid lymph nodes. No evidence of distant metastases was found on abdominal ultrasonography and chest X-ray. The patient is currently well, without evidence of disease progression one year after the second surgery. She is closely followed up undergoing clinical, radiologic and laboratory examinations every 6 months. At present, the patient is planned for fine needle aspiration biopsy; total thyroidectomy is considered as a further treatment modality [14].

\section{Conclusion}

Thyroid-type carcinoma in SO is rare. Only $6 \%$ of cases of malignant transformation in struma ovarii are bilateral and the tumors arise more commonly in the left ovary than in the right $[1,2,4]$. In the described case the tumor was unilateral on the left side. Due to the rarity of the tumor, the real incidence of malignancy, pathobiology and molecular profile of thyroid cancer arising in the ovary remain poorly studied.

Thus far only a few studies have analyzed genetic alterations in thyroid-type carcinoma in SO. Previous case series describe the presence of point mutations in BRAF, NRAS, HRAS, and RET/PTC rearrangements but none in the KRAS gene [6,15-17]. The present report therefore describes for the first time a KRAS codon 12 mutation in a case of PTC with a follicular growth pattern arising in $\mathrm{SO}$ in combination with bilateral teratoma [10-12].

The prevalence of RAS gene family mutations in thyroid-originating PTC ranges from 0 to $21 \%$ in different series (about $11 \%$ on average) $[18,19]$. The highest frequency of RAS mutations has been found in the follicular variant of PTC (43\%) while in the tumors with other morphology they are not so common [20]. Alterations most frequently involve codon 61 of HRAS and NRAS and less frequently codons 12 and 13; mutations of KRAS are very rare in general [21-23]. In line with these observations, our previous study in Serbian patients detected $R A S$ mutations in $6 / 218(2.8 \%)$ classical papillary tumors and 5/44 (11.4\%) follicular variant PTCs. Most detected mutations were in codon 61 of NRAS $(8 / 11,72.7 \%)$ and $3 / 11(17.3 \%)$ were in codon 12 of KRAS [13].

Thyroid tumors with mutant $R A S$ are frequently encapsulated and display a lower rate of nodal disease which are favorable prognostic factors. On the other hand, RAS mutations are also found in approximately one-half of poorly differentiated and anaplastic thyroid carcinomas and are associated with poor patient survival, 
suggesting that RAS may have distinct roles in the early and late stage of thyroid cancer $[19,20,24]$. Several studies have demonstrated that tumors carrying any of mutant forms of KRAS are associated with a poor prognosis in a number of human malignancies $[24,25]$. Analyzing these reports, we found that a glycine to valine mutation at codon 12 of the KRAS gene, the same mutation that we describe in this report, associates with poor survival in colon cancer [25] suggesting that this mutation may represent a marker of tumor aggressiveness, perhaps in thyroid cancer as well.

On the other hand, BRAF mutations or RET/PTC rearrangements are commonly seen in thyroid cancer arising in struma ovarii. In one of the largest and most significant studies to date, Schmidt et al., showed that every PTC in SO with the classical growth pattern had the $B R A F^{V 600 E}$ mutation, and the two mutant BRAF-harboring tumors with follicular architecture had $B R A F^{K 601 E}$ and $B R A F^{T V 599-600 M}$ mutation. No follicular carcinomas were identified in this study. Genetic alteration in benign SO has been seen in only one case in the literature. Elisei et al. recently reported the presence of RET/PTC3 rearrangement in histologically benign tissue. In carcinomas of the thyroid, numerous studies have suggested, although equivocally, that $B R A F$ mutation associates with the older age, advanced disease, classical papillary histology and poorer prognosis as indicated by disease-free and overall survival $[8,9]$. The presence of $R E T / P T C$ rearrangement correlates with some clinicopathological features of PTC such as younger age of patients, tumor morphology and a higher probability of lymph node involvement [26]. No studies have as yet correlated $R A S$ and BRAF mutational status or the presence of RET/PTC rearrangements with clinical behavior in thyroid-type carcinoma arising in SO. However, it is generally assumed that clinical behavior and prognosis of these tumors are similar to those of tumors arising in the thyroid [6,15-17].

In our study, we describe an unusual case of KRAS mutation-harboring FV-PTC in a patient with bilateral ovarian teratoma. This mutation has not been previously reported in any SO, although it is sometimes detected in PTC of the thyroid and in a variety of other tumor types. Analysis of more cases and a longer period of follow-up will be necessary to determine whether this mutation has prognostic or therapeutic value.

\section{Consent}

Written informed consent was obtained from the patient for publication of this case report and any accompanying images. A copy of the written consent is available for review by the Editor-in-Chief of this journal.

\section{Abbreviations}

SO: Struma ovarii; PTC: Papillary thyroid carcinoma; TSH: Thyroid - stimulating hormone; CA-125: Cancer antigen; PCR: Polymerase chain reaction; FVPTC: Follicular variant of papillary thyroid carcinoma; Gly: Glycine amino acid; Val: Valine amino acid.

\section{Competing interests}

We declare no conflict of interests.

\section{Acknowledgments}

This work was supported in part by Nagasaki University Global COE Program, research grant 22390189 from the JSPS to SY, and Ministry of Science and Technological Development of the Republic of Serbia Contracts No.145094 and No.173049.

\section{Author details}

'Nagasaki University Graduate School of Biomedical Sciences, 1-12-4 Sakamoto, Nagasaki, 852-8523, Japan. ${ }^{2}$ Laboratory for Radiobiology and Molecular Genetics "Vinca", Institute of Nuclear Sciences, University of Belgrade, Mike Petrovica Alasa12-14, P.O. Box 522, 11000, Belgrade, Serbia. ${ }^{3}$ University of Belgrade, School of Medicine, Dr Subotica 8, 11000, Belgrade, Serbia. Institute of Oncology and Radiology of Serbia, Surgical Oncology Clinic, Pasterova 14, 11000, Belgrade, Serbia. ${ }^{5}$ Department of Pathology, Institute for Oncology and Radiology of Serbia, Pasterova 14, 11000, Belgrade, Serbia. ${ }^{6}$ Department of Pathology, Medical Center Valjevo, 14000, Valjevo, Serbia.

\section{Authors' contributions}

The patient was examined and operated by PR and BR and these authors are responsible for the post-operative care, follow-up and clinical information. RD and MB examined the patient and reviewed the patient's files. RD will operate the patient. ZM and VK performed histopathological examination. BS and VS were responsible for the main conception and laboratory investigation. The manuscript was drafted by BS and VS, and then critically reviewed by LT, BD and SY. All authors read and approved the final manuscript.

Received: 18 January 2012 Accepted: 8 June 2012

Published: 8 June 2012

\section{References}

1. Makani S, Kim W, Gaba AR: Struma ovarii with a focus of papillary thyroid cancer: a case report and review of the literature. Gynecol Oncol 2004, 94:835-839.

2. Devaney K, Synder R, Norris H, Tavassoli F: Proliferative and histologically malignant struma ovarii: a clinicopathological study of 54 cases. Int J Gynecol Pathol 1993, 12:333-343.

3. Tavassoli F, Devilee P: World Health Organization classification of tumours. Pathology and genetics of tumours of the breast and female genital organs. Lyon: IARC Press; 2003.

4. Matsuda K, Maehama T, Kanazawa K: Malignant struma ovarii with thyrotoxicosis. Gynecol Oncol 2001, 82:575-577.

5. Nikiforov YE: Thyroid carcinoma: molecular pathways and therapeutic targets. Mod Path 2008, 21:537-643.

6. Coyne C, Nikiforov YE: RAS mutation-positive follicular variant of papillary thyroid carcinoma arising in a struma ovarii. Endocr Pathol 2010 21:144-147.

7. Kimura ET, Nikiforova MN, Zhu Z, Knauf JA, Nikiforov YE, Fagin JA: High prevalence of BRAF mutations in thyroid cancer: genetic evidence for constitutive activation of the RET/PTC-RAS-BRAF signaling pathway in papillary thyroid carcinoma. Cancer Res 2003, 63:1454-1457.

8. Xing M: BRAF mutation in thyroid cancer. Endocr Relat Cancer 2005, 12:245-262

9. Melillo RM, Castellone MD, Guarino V, De Falco V, Cirafici AM, Salvatore G, Caiazzo F, Basolo F, Giannini R, Kruhoffer M, Orntoft T, Fusco A, Santoro M: The RET/PTC-RAS-BRAF linear signaling cascade mediates the motile and mitogenic phenotype of thyroid cancer cells. J Clin Invest 2005, 115:1068-1081.

10. Nikiforov YE, Biddinger PW, Thompson LD, FASCP, FCAP: Diagnostic Pathology and Molecular Genetics of the Thyroid, A Comprehensive Guide for Practicing Thyroid Pathology:: Wolters—Kluwer; 2009:177. 
11. Volante M, Collini P, Nikiforov YE, Sakamoto A, Kakudo K, Katoh R, Lloyd RV, LiVolsi VA, Papotti M, Sobrinho-Simoes M, Bussolati G, Rosai J: Poorly differentiated thyroid carcinoma: the Turin proposal for the use of uniform diagnostic criteria and an algorithmic diagnostic approach. Am J Surg Pathol 2007, 31:1256-64.

12. DeLellis RA, Lioyd RU, Heitz PU, Eng C: World Health Organization classification of tumours. Pathology and genetics of tumours of endocrine organs. Lyon: IARC Press; 2004.

13. Stanojevic B, Dzodic R, Saenko V, Milovanovic Z, Pupic G, Zivkovic O, Markovic I, Djurisic I, Buta M, Dimitrijevic B, Rogounovitch T, Mitsutake N, Mine M, Shibata Y, Nakashima M, Yamashita S: Mutational and clinicopathological analysis of papillary thyroid carcinoma in Serbia. Endocr J 2011, 58:381-393.

14. Dzodic R, Markovic I, Inic M, Jokic N, Djurisic I, Zegarac M, Pupic G, Milovanovic Z, Jovic V, Jovanovic N: Sentinel lymph node biopsy may be used to support the decision to perform modified radical neck dissection in differentiated thyroid carcinoma. World J Surg 2006, 30:841-6.

15. Flavin R, Smyth P, Crotty P, Finn S, Cahill S, Denning K, Li J, O'Regan E, O'Leary J, Sheils O: BRAF T1799A mutation occurring in a case of malignant struma ovarii. Int J Surg Pathol 2007, 15:116-120.

16. Schmidt J, Derr V, Heinrich M, Crum C, Fletcher J, Corless C, Nose V: BRAF in papillary thyroid carcinoma of ovary (struma ovarii). Am J Surg Pathol 2007, 31:1337-1343.

17. Elisei R, Romei C, Castagna MG: RET / PTC3 rearrangement and thyroid differentiation gene analysis in a struma ovarii fortuitously revealed by elevated serum thyroglobulin concentration. Thyroid 2005, 15:1355-1361.

18. Celestino R, Magalhães J, Castro P, Triller M, Vinagre J, Soares P, SobrinhoSimões M: A follicular variant of papillary thyroid carcinoma in struma ovarii. Case report with unique molecular alterations. Histopathology 2009, 55:482-487.

19. Vasko V, Ferrand M, Di Cristofaro J, Carayon P, Henry JF, de Micco C: Specific pattern of RAS oncogene mutations in follicular thyroid tumors. J Clin Endocrinol Metab 2003, 88:2745-2752.

20. Zhu Z, Gandhi M, Nikiforova MN, Fischer AH, Nikiforov YE: Molecular profile and clinical-pathologic features of the follicular variant of papillary thyroid carcinoma. An unusually high prevalence of ras mutations. Am JClin Pathol 2003, 120:71-7.

21. Esapa CT, Johnson SJ, Kendall-Taylor P, Lennard TWJ, Harris PE: Prevalence of ras mutations in thyroid neoplasia. Clin Endocrinol (Oxf) 1999, 50:529-535

22. Kim S, Zhu X: Lessons from mouse models of thyroid cancer. Thyroid 2009, 19:1317-1331.

23. Ohori NP, Nikiforova MN, Schoedel KE, LeBeau SO, Hodak SP, Seethala RR, Carty SE, Ogilvie JB, Yip L, Nikiforov YE: Contribution of molecular testing to thyroid fine-needle aspiration cytology of "follicular lesion of undetermined significance/atypia of undetermined significance. Cancer Cytopathol 2010, 118:17-23.

24. Garcia-Rostan G, Zhao H, Camp RL, Pollan M, Herrero A, Pardo J, Wu R, Carcangiu ML, Costa J, Tallini G: Ras mutations are associated with aggressive tumor phenotypes and poor prognosis in thyroid cancer. J Clin Oncol 2003, 21:3226-3235.

25. Cerottini JP, Caplin S, Saraga E: The type of K-ras mutation determines prognosis in colorectal cancer. Am J Surg 1998, 175:198-202.

26. Elisei R, Romei C, Vorontsova T, Cosci B, Veremeychik V, Kuchinskaya E, Basolo F, Demidchik EP, Miccoli P, Pinchera A, Pacini F: RET/PTC rearrangements in thyroid nodules: studies in irradiated and not irradiated, malignant and benign thyroid lesions in children and adults. J Clin Endocrinol Metab 2001, 86:3211-3216.

doi:10.1186/1471-2407-12-224

Cite this article as: Stanojevic et al:: Unilateral follicular variant of papillary thyroid carcinoma with unique KRAS mutation in struma ovarii in bilateral ovarian teratoma: a rare case report. BMC Cancer 2012 12:224.

\section{Submit your next manuscript to BioMed Central and take full advantage of:}

- Convenient online submission

- Thorough peer review

- No space constraints or color figure charges

- Immediate publication on acceptance

- Inclusion in PubMed, CAS, Scopus and Google Scholar

- Research which is freely available for redistribution

Submit your manuscript at www.biomedcentral.com/submit
C Biomed Central 